\title{
Commutators for multilinear singular integrals on weighted Morrey spaces
}

Songbai Wang and Yinsheng Jiang*

\section{"Correspondence:} ysjiang@xju.edu.cn College of Mathematics and System Sciences, Xinjiang University,

Urumqi, 830046, P.R. China

\begin{abstract}
In this paper we study the iterated commutators for multilinear singular integrals on weighted Morrey spaces. A strong type estimate and a weak endpoint estimate for the commutators are obtained. In the last section we present a problem for the multilinear Fourier multiplier with limited smooth condition.
\end{abstract}

MSC: 42B20; 42B25

Keywords: multilinear singular integrals; multiple weights; commutators

\section{Introduction}

As an important direction of harmonic analysis, the theory of multilinear CalderónZygmund singular integral operators has attracted more and more attention, which originated from the work of Coifman and Meyer [1], and it systematically was studied by Grafakos and Torres [2, 3]. The literature of the standard theory of multilinear CalderónZygmund singular integrals is by now quite vast, for example see [2, 4-6]. In 2009, the authors [7] introduced the new multiple weights and new maximal functions and obtained some weighted estimates for multilinear Calderón-Zygmund singular integrals. They also resolved some problems opened up in [8] and [9].

Let $\mathcal{S}\left(\mathbb{R}^{n}\right)$ and $\mathcal{S}^{\prime}\left(\mathbb{R}^{n}\right)$ be the Schwartz spaces of all rapidly decreasing functions and tempered distributions, respectively. Having fixed $m \in \mathbb{N}$, let $T$ be a multilinear operator initially defined on the $m$-fold product of Schwartz spaces and taking values into the space of tempered distributions,

$$
T: \mathcal{S}\left(\mathbb{R}^{n}\right) \times \cdots \times \mathcal{S}\left(\mathbb{R}^{n}\right) \rightarrow \mathcal{S}^{\prime}\left(\mathbb{R}^{n}\right)
$$

Following [2], the $m$-multilinear Calderón-Zygmund operator $T$ satisfies the following conditions:

(S1) there exist $q_{i}<\infty(i=1, \ldots, m)$, it extends to a bounded multilinear operator from $L^{q_{1}} \times \cdots \times L^{q_{m}}$ to $L^{q}$, where $\frac{1}{q}=\frac{1}{q_{1}}+\cdots+\frac{1}{q_{m}}$;

(S2) there exists a function $K$, defined off the diagonal $x=y_{1}=\cdots=y_{m}$ in $\left(\mathbb{R}^{n}\right)^{m+1}$, satisfying

$$
T(\vec{f})(x)=T\left(f_{1}, \ldots, f_{m}\right)(x)=\int_{\left(\mathbb{R}^{m}\right)^{n}} K\left(x, y_{1}, \ldots, y_{m}\right) f_{1}\left(y_{1}\right) \cdots f_{m}\left(y_{m}\right) d y_{1} \cdots d y_{m}
$$

○2014 Wang and Jiang; licensee Springer. This is an Open Access article distributed under the terms of the Creative Commons Attribution License (http://creativecommons.org/licenses/by/2.0), which permits unrestricted use, distribution, and reproduction in any medium, provided the original work is properly cited. 
for all $x \notin \bigcap_{j=1}^{m} \operatorname{supp} f_{j}$ and $f_{1}, \ldots, f_{m} \in \mathcal{S}\left(\mathbb{R}^{n}\right)$, where

$$
\left|K\left(y_{0}, y_{1}, \ldots, y_{m}\right)\right| \leq \frac{A}{\left(\sum_{l, k=0}^{m}\left|y_{l}-y_{k}\right|\right)^{m n}}
$$

and

$$
\left|K\left(y_{0}, \ldots, y_{j}, \ldots, y_{m}\right)-K\left(y_{0}, \ldots, y_{j}^{\prime}, \ldots, y_{m}\right)\right| \leq \frac{A\left|y_{j}-y_{j}^{\prime}\right|^{\epsilon}}{\left(\sum_{l, k=0}^{m}\left|y_{l}-y_{k}\right|\right)^{m n+\epsilon}}
$$

for some $\epsilon>0$ and all $0 \leq j \leq m$, whenever $\left|y_{j}-y_{j}^{\prime}\right| \leq \frac{1}{2} \max _{0 \leq k \leq m}\left|y_{j}-y_{k}\right|$.

We also use some notation following [10]. Given a locally integrable vector function $\mathbf{b}=\left(b_{1}, \ldots, b_{m}\right) \in(B M O)^{m}$, the commutator of $\mathbf{b}$ and the $m$-linear Calderón-Zygmund operator $T$, denoted here by $T_{\Sigma \mathbf{b}}$, was introduced by Pérez and Torres in [9] and is defined via

$$
T_{\Sigma \mathbf{b}}(\vec{f})=\sum_{j=1}^{m} T_{b_{j}}^{j}(\vec{f})
$$

where

$$
T_{b_{j}}^{j}(\vec{f})=b_{j} T(\vec{f})-T\left(f_{1}, \ldots, b_{j} f_{j}, \ldots, f_{N}\right)
$$

The iterated commutator $T_{\Pi \mathbf{b}}$ is defined by

$$
T_{\Pi \mathbf{b}}(\vec{f})=\left[b_{1}, \ldots,\left[b_{m-1},\left[b_{m}, T\right]_{m}\right]_{m-1} \cdots\right]_{1}(\vec{f})
$$

To clarify the notations, if $T$ is associated in the usual way with a Calderón-Zygmund kernel $K$, then at a formal level

$$
T_{\Sigma \mathbf{b}}(\vec{f})(x)=\int_{\left(\mathbb{R}^{n}\right)^{m}} \sum_{j=1}^{m}\left(b_{j}(x)-b_{j}\left(y_{j}\right)\right) K\left(x, y_{1}, \ldots, y_{m}\right) f_{1}\left(y_{1}\right) \cdots f_{m}\left(y_{m}\right) d y_{1} \cdots d y_{m}
$$

and

$$
T_{\Pi \mathbf{b}}(\vec{f})(x)=\int_{\left(\mathbb{R}^{n}\right)^{m}} \prod_{j=1}^{m}\left(b_{j}(x)-b_{j}\left(y_{j}\right)\right) K\left(x, y_{1}, \ldots, y_{m}\right) f_{1}\left(y_{1}\right) \cdots f_{m}\left(y_{m}\right) d y_{1} \cdots d y_{m}
$$

It was shown in [2] that if $\frac{1}{q}=\frac{1}{q_{1}}+\cdots+\frac{1}{q_{m}}$, then an $m$-linear Calderón-Zygmund operator $T$ maps from $L^{q_{1}} \times \cdots \times L^{q_{m}}$ to $L^{q}$, when $1<q_{j}<\infty$ for all $j=1, \ldots, m$; and from $L^{q_{1}} \times$ $\cdots \times L^{q_{m}}$ to $L^{q, \infty}$, when $1 \leq q_{j}<\infty$ for all $j=1, \ldots, m$, and $\min _{1 \leq j \leq m} q_{j}=1$. The weighted strong and weak $L^{q}$ boundedness of $T$ is also true for weights in the class $A_{\vec{P}}$ which will be introduced in next section (see Corollary 3.9 [7]). It was proved in [9] that $T_{\Sigma \mathbf{b}}$ is bounded from $L^{q_{1}} \times \cdots \times L^{q_{m}}$ to $L^{q}$ for all indices satisfying $\frac{1}{q}=\frac{1}{q_{1}}+\cdots+\frac{1}{q_{m}}$ with $q>1$ and $q_{j}>1$, $j=1, \ldots, m$. The result was extended in [7] to all $q>1 / m$. In fact, the authors obtained the weighted $L^{q}$-version bounds as follows, for all $\vec{\omega} \in A_{\vec{P}}$ :

$$
\left\|T_{\Sigma \mathbf{b}}(\vec{f})\right\|_{L^{q\left(v_{\vec{\omega}}\right)}} \leq C\|\vec{b}\|_{B M O^{m}} \prod_{j=1}^{m}\left\|f_{j}\right\|_{L^{q_{j}\left(\omega_{j}\right)}}
$$


As may be expected from the situation in the linear case, $T_{\Sigma \mathbf{b}}$ is not bounded from $L^{1} \times$ $\cdots \times L^{1}$ to $L^{1, \infty}$. However, a sharp weak-type estimate in a very general sense was obtained in [7], that is, for all $\vec{\omega} \in A_{(1, \ldots, 1)}$,

$$
v_{\vec{\omega}}\left\{x \in \mathbb{R}^{n}:\left|T_{\Sigma \mathbf{b}}(\vec{f})(x)\right|>t^{m}\right\} \leq C \prod_{j=1}^{m}\left(\int_{\mathbb{R}^{n}} \Phi\left(\frac{\left|f_{j}(x)\right|}{t}\right) \omega_{j}(x) d x\right)^{1 / m},
$$

where $\Phi(t)=t\left(1+\log ^{+} t\right)$. When $m=1$, the above endpoint estimate was obtained in [11]. The same as for $T_{\Sigma \mathbf{b}}$, the strong type bound and the endpoint estimate for $T_{\Pi \mathbf{b}}$ were also established by Pérez et al. in [10].

The weighted Morrey spaces $L^{p, k}(w)$ was introduced by Komori and Shirai [6]. Moreover, they showed that some classical integral operators and corresponding commutators are bounded in weighted Morrey spaces. Some other authors have been interested in this space for sublinear operators, see [12-14]. In [15], Ye proved two results similar to Pérez and Trujillo-González [11] for the multilinear commutators of the normal CalderónZygmund operators on weighted Morrey spaces. Wang and Yi [16] considered the multilinear Calderón-Zygmund operators on weighted Morrey spaces and obtained some results similar to weighted Lebesgue spaces.

We will prove the following strong type bound for $T_{\Pi \mathbf{b}}$ on weighted Morrey spaces.

Theorem 1.1 Let $T$ be an m-linear Calderón-Zygmund operator; $\vec{\omega} \in A_{\vec{P}} \cap\left(A_{\infty}\right)^{m}$ with

$$
\frac{1}{p}=\frac{1}{p_{1}}+\cdots+\frac{1}{p_{m}}
$$

and $1<p_{j}<\infty, j=1, \ldots, m$; and $\mathbf{b} \in B M O^{m}$. Then, for any $0<k<1$, there exists a constant C such that

$$
\left\|T_{\Pi \mathbf{b}}(\vec{f})\right\|_{L^{p, k}\left(v_{\bar{\omega}}\right)} \leq C \prod_{j=1}^{m}\left\|b_{j}\right\|_{B M O} \prod_{j=1}^{m}\left\|f_{j}\right\|_{L^{p_{j}, k}\left(\omega_{j}\right)} .
$$

The following endpoint estimate will also be proved.

Theorem 1.2 Let $T$ be an m-linear Calderón-Zygmund operator; $0<k<1, \vec{\omega} \in A_{(1, \ldots, 1)} \cap$ $\left(A_{\infty}\right)^{m}$, and $\mathbf{b} \in B M O^{m}$. Then, for any $\lambda>0$ and cube $Q$, there exists a constant $C$ such that

$$
\frac{1}{v_{\vec{\omega}}(Q)^{k}} v_{\vec{\omega}}\left\{x \in Q:\left|T_{\Pi \mathbf{b}}(\vec{f})(x)\right|>\lambda\right\} \leq C \prod_{j=1}^{m}\left[\left\|f_{j} / \lambda\right\|_{L^{\Phi}(m), k\left(\omega_{j}\right)}\right]^{1 / m},
$$

where $\Phi^{(m)}=\overbrace{\Phi \circ \cdots \circ \Phi}^{m}, \Phi(t)=t\left(1+\log ^{+} t\right)$ and $\|f\|_{L^{\Phi}(m), k(\omega)}=\left\|\Phi^{(m)}(|f|)\right\|_{L^{1, k}(\omega)}$.

Remark 1.1 Here we remark that the above estimate is also valid for $T_{\Sigma \mathbf{b}}$.

\section{Some definitions and results}

In this section, we introduce some definitions and results used later.

Definition 2.1 ( $A_{p}$ weights) A weight $\omega$ is a nonnegative, locally integrable function on $\mathbb{R}^{n}$. Let $1<p<\infty$, a weight function $\omega$ is said to belong to the class $A_{p}$, if there is a 
constant $C$ such that for any cube $Q$,

$$
\left(\frac{1}{|Q|} \int_{Q} \omega(x) d x\right)\left(\frac{1}{|Q|} \int_{Q} \omega(x)^{1-p^{\prime}} d x\right)^{p-1} \leq C
$$

and to the class $A_{1}$, if there is a constant $C$ such that for any cube $Q$,

$$
\frac{1}{|Q|} \int_{Q} \omega(x) d x \leq C \inf _{x \in Q} \omega(x)
$$

We denote $A_{\infty}=\bigcup_{p>1} A_{p}$.

Definition 2.2 (Multiple weights) For $m$ exponents $p_{1}, \ldots, p_{m} \in[1, \infty)$, we often write $p$ for the number given by $p=\sum_{j=1}^{m} p_{j}$ and denote by $\vec{P}$ the vector $\left(p_{1}, \ldots, p_{m}\right)$. A multiple weight $\vec{\omega}=\left(\omega_{1}, \ldots, \omega_{m}\right)$ is said to satisfy the $A_{\vec{P}}$ condition if for

$$
v_{\vec{\omega}}=\prod_{j=1}^{m} \omega^{p / p_{j}}
$$

we have

$$
\sup _{Q}\left(\frac{1}{|Q|} \int_{Q} v_{\vec{\omega}}(x) d x\right)^{1 / p} \prod_{j=1}^{m}\left(\frac{1}{|Q|} \int_{Q} \omega_{j}(x)^{1-p_{j}^{\prime}} d x\right)^{1 / p_{j}^{\prime}}<\infty
$$

when $p_{j}=1,\left(\frac{1}{|Q|} \int_{Q} \omega_{j}(x)^{1-p_{j}^{\prime}} d x\right)^{1 / p_{j}^{\prime}}$ is understood as $\left(\inf _{x} \omega(x)\right)^{-1}$. As remarked in [7], $\prod_{j=1}^{m} A_{p_{j}}$ is strictly contained in $A_{\vec{P}}$, moreover, in general $\vec{\omega} \in A_{\vec{P}}$ does not imply $\omega_{j} \in L_{\text {loc }}^{1}$ for any $j$, but instead

$$
\vec{\omega} \in A_{\vec{p}} \Leftrightarrow\left\{\begin{array}{l}
\left(v_{\vec{\omega}}\right)^{p} \in A_{m p}, \\
1-p_{j}^{\prime} \in A_{m p_{j}^{\prime}}, \quad j=1, \ldots, m, \\
\omega_{j},
\end{array}\right.
$$

where the condition $\omega_{j}^{1-p_{j}^{\prime}} \in A_{m p_{j}^{\prime}}$ in the case $p_{j}=1$ is understood as $\omega_{j}^{1 / m} \in A_{1}$.

Definition 2.3 (Weighted Morrey spaces) Let $0<p<\infty, 0<k<1$, and $\omega$ be a weight function on $\mathbb{R}^{n}$. The weighted Morrey space is defined by

$$
L^{p, k}(\omega)=\left\{f \in L_{\mathrm{loc}}^{p}:\|f\|_{L^{p, k}(\omega)}<\infty\right\},
$$

where

$$
\|f\|_{L^{p, k}(\omega)}=\sup _{Q}\left(\frac{1}{\omega(Q)^{k}} \int_{Q}|f(x)|^{p} \omega(x)\right)^{1 / p} .
$$

The weighted weak Morrey space is defined by

$$
W L^{p, k}(\omega)=\left\{f \text { measurable }:\|f\|_{W L^{p, k}(\omega)}<\infty\right\},
$$


where

$$
\|f\|_{W L^{p, k}(\omega)}=\sup _{Q} \inf _{\lambda>0} \frac{\lambda}{\omega(Q)^{k / p}} \omega(\{x \in Q:|f|(x)>\lambda\})^{1 / p} .
$$

Definition 2.4 (Maximal function) For $\Phi(t)=t\left(1+\log ^{+} t\right)$ and a cube $Q$ in $\mathbb{R}^{n}$ we will consider the average $\|f\|_{\Phi, Q}$ of a function $f$ given by the Luxemburg norm

$$
\|f\|_{\Phi, Q}=\inf \left\{\lambda>0: \frac{1}{|Q|} \int_{Q} \Phi\left(\frac{|f(x)|}{\lambda}\right) d x \leq 1\right\}
$$

and the corresponding maximal is naturally defined by

$$
M_{\Phi} f(x)=\sup _{Q \ni x}\|f\|_{\Phi, Q}
$$

and the multilinear maximal operator $\mathcal{M}_{\Phi, Q}$ is given by

$$
\mathcal{M}_{\Phi}(\vec{f})(x)=\sup _{Q \ni x} \prod_{j=1}^{m}\left\|f_{j}\right\|_{\Phi, Q}
$$

The following pointwise equivalence is very useful:

$$
M_{\Phi} f(x) \approx M^{2} f(x)
$$

where $M$ is the Hardy-Littlewood maximal function. We refer reader to $[7,10]$ and their references for details.

We say that a weight $\omega$ satisfies the doubling condition, simply denoted $\omega \in \Delta_{2}$, if there is a constant $C>0$ such that $\omega(2 Q) \leq C \omega(Q)$ holds for any cube $Q$. If $\omega \in A_{p}$ with $1 \leq p<\infty$, we know that $\omega(\lambda Q) \leq \lambda^{n p}[\omega]_{A_{p}} \omega(Q)$ for all $\lambda>1$; then $\omega \in \Delta_{2}$.

Lemma 2.1 ([6]) Suppose $\omega \in \Delta_{2}$, then there exists a constant $D>1$ such that

$$
\omega(2 Q) \geq D \omega(Q)
$$

for any cube.

Lemma 2.2 ([16]) If $\omega_{j} \in A_{\infty}$, then for any cube $Q$, we have

$$
\int_{Q} \prod_{j=1}^{m} \omega_{j}^{\theta_{j}}(x) d x \geq \prod_{j=1}^{m}\left(\frac{\int_{Q} \omega_{j}(x) d x}{\left[\omega_{j}\right]_{\infty}}\right)^{\theta_{j}}
$$

where $\sum_{j=1}^{m} \theta_{j}=1,0 \leq \theta_{j} \leq 1$.

Lemma 2.3 ([17]) Suppose $\omega \in A_{\infty}$, then $\|b\|_{B M O(\omega)} \approx\|b\|_{B M O}$. Here

$$
B M O(\omega)=\left\{b:\|b\|_{B M O(\omega)}=\sup _{Q} \frac{1}{\omega(Q)} \int_{Q}\left|b(x)-b_{Q, \omega}\right| \omega(x) d x<\infty\right\},
$$

and $b_{Q, \omega}=\frac{1}{\omega(Q)} \int_{Q} b(x) \omega(x) d x$. 
From the fact $\left|b_{2^{j} Q}-b_{Q}\right| \leq C j\|b\|_{B M O}$ and Lemma 2.3, we deduce that $\left|b_{2^{j}, \omega}-b_{Q, \omega}\right| \leq$ $C j\|b\|_{B M O}$. The following lemma is the multilinear version of the Fefferman-Stein type inequality.

Lemma 2.4 (Theorem 3.12 [7]) Assume that $\omega_{i}$ is a weight in $A_{1}$ for all $i=1, \ldots, m$, and set $v=\left(\prod_{i=1}^{m} \omega_{i}\right)^{1 / m}$. Then

$$
\left\|\prod_{j=1}^{m} M\left(f_{j}\right)\right\|_{L^{p, \infty}(v)} \leq \prod_{j=1}^{m}\left\|f_{j}\right\|_{L^{1}\left(M \omega_{j}\right)} .
$$

Lemma 2.5 (Proposition $3.13[7])$ Let $\frac{1}{p}=\frac{1}{p_{1}}+\cdots+\frac{1}{p_{m}}$. If $1 \leq p_{j} \leq \infty, j=1, \ldots, m$, then

$$
\|\mathcal{M}(\vec{f})\|_{L^{p, \infty}\left(v_{\vec{\omega}}\right)} \leq \prod_{j=1}^{m}\left\|f_{j}\right\|_{L^{p_{j}}\left(M \omega_{j}\right)} \cdot
$$

Lemma 2.6 (Theorem $3.2[10]$ ) Let $p>0$ and let $\omega$ be a weight in $A_{\infty}$. Suppose that $\mathbf{b} \in$ $B M O^{m}$. Then there exist $C_{\omega}$ (independent of $\mathbf{b}$ ) and $C_{\omega, \mathbf{b}}$ such that

$$
\int_{\mathbb{R}^{n}}\left|T_{\Pi \mathbf{b}}(\vec{f})(x)\right| \omega(x) d x \leq C_{\omega} \prod_{j=1}^{m}\left\|b_{j}\right\|_{B M O} \int_{\mathbb{R}^{n}} \mathcal{M}_{\Phi}(\vec{f})(x)^{p} \omega(x) d x
$$

and

$$
\begin{aligned}
& \sup _{t>0} \frac{1}{\Phi^{(m)}\left(\frac{1}{t}\right)} \omega\left(\left\{y \in \mathbb{R}^{n}:\left|T_{\Pi \mathbf{b}}(\vec{f})(y)\right|>t^{m}\right\}\right) \\
& \quad \leq C_{\omega, \mathbf{b}} \sup _{t>0} \frac{1}{\Phi^{(m)}\left(\frac{1}{t}\right)} \omega\left(\left\{y \in \mathbb{R}^{n}:\left|\mathcal{M}_{\Phi}(\vec{f})(y)\right|>t^{m}\right\}\right)
\end{aligned}
$$

for all $\vec{f}=\left(f_{1}, \ldots, f_{m}\right)$ bounded with compact support.

Lemma 2.7 (Theorem $4.1[10])$ Let $\omega \in A_{(1, \ldots, 1)}$. Then there exists a constant $C$ such that

$$
v_{\vec{\omega}}\left(\left\{x \in \mathbb{R}^{n}:\left|\mathcal{M}_{L \log L}(\vec{f})(x)\right|>t^{m}\right\}\right) \leq C \prod_{j=1}^{m}\left(\int_{\mathbb{R}^{n}} \Phi^{(m)}\left(\frac{\left|f_{j}(x)\right|}{t}\right) \omega_{j}(x) d x\right)^{1 / m} .
$$

By the above two inequalities, Pérez and Trujillo-González obtained the following results.

Lemma 2.8 (Theorem $1.1[10]$ ) Let $T$ be an m-linear Calderón-Zygmund operator; $\vec{\omega} \in A_{\vec{P}}$ with

$$
\frac{1}{p}=\frac{1}{p_{1}}+\cdots+\frac{1}{p_{m}}
$$

and $1<p_{j}<\infty, j=1, \ldots, m ;$ and $\mathbf{b} \in B M O^{m}$. Then there exists a constant $C$ such that

$$
\left\|T_{\Pi \mathbf{b}}(\vec{f})\right\|_{L^{p}\left(v_{\vec{\omega}}\right)} \leq C \prod_{j=1}^{m}\left\|b_{j}\right\|_{B M O} \prod_{j=1}^{m}\left\|f_{j}\right\|_{L^{p_{j}}\left(\omega_{j}\right)} .
$$


Lemma 2.9 (Theorem 1.2 [10]) Let $T$ be an m-linear Calderón-Zygmund operator; $\vec{\omega} \in$ $A_{(1, \ldots, 1)}$, and $\mathbf{b} \in B M O^{m}$. Then, for any $\lambda>0$ and cube $Q$, there exists a constant $C$ such that

$$
\nu_{\vec{\omega}}\left\{x \in \mathbb{R}^{n}:\left|T_{\Pi \mathbf{b}}(\vec{f})(x)\right|>\lambda\right\} \leq C \prod_{j=1}^{m}\left(\int_{\mathbb{R}^{n}} \Phi^{(m)}\left(\frac{\left|f_{j}(x)\right|}{t}\right) \omega_{j}(x) d x\right)^{1 / m},
$$

where $\Phi(t)=t\left(1+\log ^{+} t\right)$ and $\Phi^{(m)}=\overbrace{\Phi \circ \cdots \circ \Phi}^{m}$.

\section{Proofs of theorems}

We only present the case $m=2$ for simplicity, but, as the reader will immediately notice, a complicated notation and a similar procedure can be followed to obtain the general case. Our arguments will be standard.

Proof of Theorem 1.1 For any cube $Q$, we split $f_{j}$ into $f_{j}^{0}+f_{j}^{\infty}$, where $f_{j}^{0}=f_{j} \chi_{2 Q}$ and $f_{j}^{\infty}=$ $f_{j}-f_{j}^{0}, j=1,2$. Then we only need to verify the following inequalities:

$$
\begin{aligned}
& I=\left(\frac{1}{v_{\vec{\omega}}(Q)^{k}} \int_{Q}\left|T_{\Pi \mathbf{b}}\left(f_{1}^{0}, f_{2}^{0}\right)(x)\right|^{p} v_{\vec{\omega}}(x) d x\right)^{1 / p} \leq C \prod_{j=1}^{2}\left\|b_{j}\right\|_{B M O} \prod_{j=1}^{2}\left\|f_{j}\right\|_{L^{p_{j}, k}\left(\omega_{j}\right)}, \\
& I I=\left(\frac{1}{v_{\vec{\omega}}(Q)^{k}} \int_{Q}\left|T_{\Pi \mathbf{b}}\left(f_{1}^{0}, f_{2}^{\infty}\right)(x)\right|^{p} v_{\vec{\omega}}(x) d x\right)^{1 / p} \leq C \prod_{j=1}^{2}\left\|b_{j}\right\|_{B M O} \prod_{j=1}^{2}\left\|f_{j}\right\|_{L^{p_{j}, k}\left(\omega_{j}\right)}, \\
& I I I=\left(\frac{1}{v_{\vec{\omega}}(Q)^{k}} \int_{Q}\left|T_{\Pi \mathbf{b}}\left(f_{1}^{\infty}, f_{2}^{0}\right)(x)\right|^{p} v_{\vec{\omega}}(x) d x\right)^{1 / p} \leq C \prod_{j=1}^{2}\left\|b_{j}\right\|_{B M O} \prod_{j=1}^{2}\left\|f_{j}\right\|_{L^{p_{j}, k}\left(\omega_{j}\right)}, \\
& I V=\left(\frac{1}{v_{\vec{\omega}}(Q)^{k}} \int_{Q}\left|T_{\Pi \mathbf{b}}\left(f_{1}^{\infty}, f_{2}^{\infty}\right)(x)\right|^{p} v_{\vec{\omega}}(x) d x\right)^{1 / p} \leq C \prod_{j=1}^{2}\left\|b_{j}\right\|_{B M O} \prod_{j=1}^{2}\left\|f_{j}\right\|_{L^{p_{j}, k}}{\left(\omega_{j}\right)^{*}}^{.}
\end{aligned}
$$

From Lemma 2.8 and Lemma 2.2, we get

$$
\begin{aligned}
I & \leq C \frac{1}{v_{\vec{\omega}}(Q)^{k / p}} \prod_{j=1}^{2}\left\|b_{j}\right\|_{B M O}\left(\int_{\mathbb{R}^{n}}\left|f_{j}^{0}(x)\right|^{p_{j}} \omega_{j}(x) d x\right)^{1 / p_{j}} \\
& \leq C \frac{1}{v_{\vec{\omega}}(Q)^{k / p}} \prod_{j=1}^{2}\left[\left\|b_{j}\right\|_{B M O} \omega_{j}(2 Q)^{k / p_{j}}\left\|f_{j}\right\|_{L^{p_{j}, k}\left(\omega_{j}\right)}\right] \\
& \leq C \prod_{j=1}^{2}\left[\left\|b_{j}\right\|_{B M O}\left\|f_{j}\right\|_{L^{p_{j}, k}\left(\omega_{j}\right)}\right] .
\end{aligned}
$$

Since $I I$ and $I I I$ are symmetric we only estimate $I I$. Taking $\lambda_{j}=\left(b_{j}\right)_{Q, \omega_{j}}$, the operator $T_{\Pi \mathbf{b}}$ can be divided into four parts:

$$
\begin{aligned}
T_{\text {пь }} & \left(f_{1}^{0}, f_{2}^{\infty}\right)(x) \\
= & \left(b_{1}(x)-\lambda_{1}\right)\left(b_{2}(x)-\lambda_{2}\right) T\left(f_{1}^{0}, f_{2}^{\infty}\right)(x)-\left(b_{1}(x)-\lambda_{1}\right) T\left(f_{1}^{0},\left(b_{2}-\lambda_{2}\right) f_{2}^{\infty}\right)(x) \\
& -\left(b_{2}(x)-\lambda_{2}\right) T\left(\left(b_{1}-\lambda_{1}\right) f_{1}^{0}, f_{2}^{\infty}\right)(x)+T\left(\left(b_{1}-\lambda_{1}\right) f_{1}^{0},\left(b_{2}-\lambda_{2}\right) f_{2}^{\infty}\right)(x) \\
= & I I_{1}+I I_{2}+I I_{3}+I I_{4} .
\end{aligned}
$$


Using the size condition (2) of $K$, Definition 2.2, and Lemma 2.2, we deduce that for any $x \in Q$,

$$
\begin{aligned}
& \left|T\left(f_{1}^{0}, f_{2}^{\infty}\right)(x)\right| \\
& \leq C \int_{2 Q} \int_{\mathbb{R}^{n} \backslash 2 Q} \frac{\left|f_{1}\left(y_{1}\right) f_{2}\left(y_{2}\right)\right|}{\left(\left|x-y_{1}\right|+\left|x-y_{2}\right|\right)^{2 n}} d y_{2} d y_{1} \\
& \leq C \int_{2 Q}\left|f_{1}\left(y_{1}\right)\right| d y_{1} \sum_{l=1}^{\infty} \frac{1}{\left|2^{l} Q\right|^{2}} \int_{2^{l+1} Q \backslash 2^{l} Q}\left|f_{2}\left(y_{2}\right)\right| d y_{2} \\
& \leq C \sum_{l=1}^{\infty} \prod_{j=1}^{2} \frac{1}{\left|2^{l+1} Q\right|} \int_{2^{l+1} Q}\left|f_{j}\left(y_{j}\right)\right| d y_{j} \\
& \leq C \sum_{l=1}^{\infty} \prod_{j=1}^{2} \frac{1}{\left|2^{l+1} Q\right|}\left(\int_{2^{l+1} Q}\left|f_{j}\left(y_{j}\right)\right|^{p_{j}} \omega_{j}\left(y_{j}\right) d y_{j}\right)^{1 / p_{j}} \\
& \times\left(\int_{2^{l+1} Q} \omega_{j}\left(y_{j}\right)^{1-p_{j}^{\prime}} d y_{j}\right)^{1 / p_{j}^{\prime}} \\
& \leq C \sum_{l=1}^{\infty} \frac{1}{\left|2^{l+1} Q\right|^{2}} \frac{\left|2^{l+1} Q\right|^{\frac{1}{p}+\frac{1}{p_{1}^{\prime}}+\frac{1}{p_{2}^{\prime}}}}{v_{\vec{\omega}\left(2^{l+1} Q\right)}^{2}} \prod_{j=1}^{2}\left\|f_{j}\right\|_{L^{p_{j}, k}\left(\omega_{j}\right)} \omega_{j}\left(2^{l+1} Q\right)^{k / p_{j}} \\
& \leq C \prod_{j=1}^{2}\left\|f_{j}\right\|_{L^{p_{j}, k}}\left(\omega_{j}\right) \sum_{l=1}^{\infty} v_{\vec{\omega}}\left(2^{l+1} Q\right)^{(k-1) / p} .
\end{aligned}
$$

Taking the above estimate together with Hölder's inequality and Lemma 2.3, we have

$$
\begin{aligned}
&\left(\frac{1}{v_{\vec{\omega}}(Q)^{k}} \int_{Q}\left|I I_{1}\right|^{p} v_{\vec{\omega}}(x) d x\right)^{1 / p} \\
& \leq \frac{1}{v_{\vec{\omega}}(Q)^{k / p}}\left(\int_{Q}\left|\left(b_{1}(x)-\lambda_{1}\right)\left(b_{2}(x)-\lambda_{2}\right)\right|^{p} v_{\vec{\omega}}(x) d x\right)^{1 / p} \\
& \times \prod_{j=1}^{2}\left\|f_{j}\right\|_{L^{p_{j}, k}} \sum_{l=1}^{\infty} v_{\vec{\omega}}\left(2^{l+1} Q\right)^{(k-1) / p} \\
& \leq \frac{v_{\vec{\omega}}(Q)^{1 / p}}{v_{\vec{\omega}}(Q)^{k / p}} \prod_{j=1}^{2}\left(\frac{1}{v_{\vec{\omega}}(Q)} \int_{Q}\left|\left(b_{j}(x)-\lambda_{1}\right)\right|^{2 p} v_{\vec{\omega}}(x) d x\right)^{1 / 2 p} \\
& \quad \times \prod_{j=1}^{2}\left\|f_{j}\right\|_{L^{p_{j}, k}} \sum_{l=1}^{\infty} v_{\vec{\omega}}\left(2^{l+1} Q\right)^{(k-1) / p} \\
& \leq \prod_{j=1}^{2}\left\|b_{j}\right\|_{B M O}\left\|f_{j}\right\|_{L^{p_{j}, k}\left(\omega_{j}\right)^{\prime}}
\end{aligned}
$$

where the last inequality is obtained by the property of $A_{\infty}$ : there is a constant $\delta>0$ such that

$$
\frac{v_{\vec{\omega}}(Q)}{v_{\vec{\omega}}\left(2^{l+1} Q\right)} \leq C\left(\frac{|Q|}{\left|2^{l+1} Q\right|}\right)^{\delta}
$$


For $I I_{2}$, from the size condition (2) of $K$, the $A_{\vec{P}}$ condition, Lemma 2.2, and Lemma 2.3, it follows that

$$
\begin{aligned}
& \left|T\left(f_{1}^{0},\left(b_{2}-\lambda_{2}\right) f_{2}^{\infty}\right)(x)\right| \\
& \leq C \int_{2 Q}\left|f_{1}\left(y_{1}\right)\right| d y_{1} \sum_{l=1}^{\infty} \frac{1}{\left|2^{l} Q\right|^{2}} \int_{2^{l+1} Q \backslash 2^{l} Q}\left|\left(b_{2}\left(y_{2}\right)-\lambda_{2}\right) f_{2}\left(y_{2}\right)\right| d y_{2} \\
& \leq C \sum_{l=1}^{\infty} \frac{1}{\left|2^{l+1} Q\right|^{2}}\left(\int_{2^{l+1} Q}\left|f_{1}\left(y_{1}\right)\right|^{p_{1}} \omega_{j}\left(y_{1}\right) d y_{1}\right)^{1 / p_{1}}\left(\int_{2^{l+1} Q} \omega_{1}\left(y_{1}\right)^{1-p_{1}^{\prime}} d y_{j}\right)^{1 / p_{1}^{\prime}} \\
& \times\left(\int_{2^{l+1} Q}\left|f_{2}\left(y_{2}\right)\right|^{p_{2}} \omega_{2}\left(y_{2}\right) d y_{2}\right)^{1 / p_{2}} \\
& \times\left(\int_{2^{l+1} Q}\left|b_{2}\left(y_{2}\right)-\lambda_{2}\right|^{p_{2}^{\prime}} \omega_{2}\left(y_{2}\right)^{-p_{2}^{\prime} / p_{2}} d y_{2}\right)^{1 / p_{2}^{\prime}} \\
& \leq C \sum_{l=1}^{\infty} l \prod_{j=1}^{2} \frac{1}{\left|2^{l+1} Q\right|}\left(\int_{2^{l+1} Q}\left|f_{j}\left(y_{j}\right)\right|^{p_{j}} \omega_{j}\left(y_{j}\right) d y_{j}\right)^{1 / p_{j}}\left(\int_{2^{l+1} Q} \omega_{j}\left(y_{j}\right)^{1-p_{j}^{\prime}} d y_{j}\right)^{1 / p_{j}^{\prime}} \\
& \leq C \prod_{j=1}^{2}\left\|f_{j}\right\|_{L^{p_{j}, k}\left(\omega_{j}\right)} \sum_{l=1}^{\infty} l v_{\vec{\omega}}\left(2^{l+1} Q\right)^{(k-1) / p} .
\end{aligned}
$$

The third inequality can be deduced by the fact that

$$
\left(\frac{1}{\omega\left(2^{j+1} Q\right)} \int_{2^{l+1} Q}\left|b(y)-b_{Q, \omega}\right|^{p} \omega(y) d y\right)^{1 / p} \leq C l\|b\|_{B M O(\omega)} .
$$

Hölder's inequality and Lemma 2.3 tell us

$$
\begin{aligned}
& \left(\frac{1}{v_{\vec{\omega}}(Q)^{k}} \int_{Q}\left|I I_{2}\right|^{p} v_{\vec{\omega}}(x) d x\right)^{1 / p} \\
& \quad \leq C \frac{1}{v_{\vec{\omega}}(Q)^{k / p}}\left(\int_{Q}\left|\left(b_{1}(x)-\lambda_{1}\right)\right|^{p} v_{\vec{\omega}}(x) d x\right)^{1 / p} \prod_{j=1}^{2}\left\|f_{j}\right\|_{L^{p_{j}, k}} \sum_{l=1}^{\infty} l v_{\vec{\omega}}\left(2^{l+1} Q\right)^{(k-1) / p} \\
& \quad \leq C \frac{v_{\vec{\omega}}(Q)^{1 / p}}{v_{\vec{\omega}}(Q)^{k / p}} \prod_{j=1}^{2}\left\|f_{j}\right\|_{L^{p_{j}, k}} \sum_{l=1}^{\infty} l v_{\vec{\omega}}\left(2^{l+1} Q\right)^{(k-1) / p} \\
& \quad \leq C \prod_{j=1}^{2}\left\|b_{j}\right\|_{B M O}\left\|f_{j}\right\|_{L^{p_{j}, k}\left(\omega_{j}\right)} .
\end{aligned}
$$

Similarly, we get

$$
\begin{aligned}
& \left|T\left(f_{1}^{0},\left(b_{2}-\lambda_{2}\right) f_{2}^{\infty}\right)(x)\right| \\
& \leq C \sum_{l=1}^{\infty} \frac{1}{\left|2^{l+1} Q\right|^{2}}\left(\int_{2^{l+1} Q}\left|f_{1}\left(y_{1}\right)\right|^{p_{1}} \omega_{j}\left(y_{1}\right) d y_{1}\right)^{1 / p_{1}} \\
& \quad \times\left(\int_{2^{l+1} Q}\left|b_{1}\left(y_{1}\right)-\lambda_{1}\right|^{p_{1}^{\prime}} \omega_{1}\left(y_{1}\right)^{1-p_{1}^{\prime}} d y_{j}\right)^{1 / p_{1}^{\prime}}
\end{aligned}
$$




$$
\begin{aligned}
& \times\left(\int_{2^{l+1} Q}\left|f_{2}\left(y_{2}\right)\right|^{p_{2}} \omega_{2}\left(y_{2}\right) d y_{2}\right)^{1 / p_{2}}\left(\int_{2^{l+1} Q} \omega_{2}\left(y_{2}\right)^{-p_{2}^{\prime} / p_{2}} d y_{2}\right)^{1 / p_{2}^{\prime}} \\
\leq & C \prod_{j=1}^{2}\left\|f_{j}\right\|_{L^{p_{j}, k}\left(\omega_{j}\right)} \sum_{l=1}^{\infty} l v_{\vec{\omega}}\left(2^{l+1} Q\right)^{(k-1) / p},
\end{aligned}
$$

and so

$$
\left(\frac{1}{v_{\vec{\omega}}(Q)^{k}} \int_{Q}\left|I I_{3}\right|^{p} v_{\vec{\omega}}(x) d x\right)^{1 / p} \leq C \prod_{j=1}^{2}\left\|b_{j}\right\|_{B M O}\left\|f_{j}\right\|_{L^{p_{j}, k}\left(\omega_{j}\right)} .
$$

The term $I_{4}$ is estimated in a similar way and we deduce

$$
\begin{aligned}
& \left|T\left(\left(b_{1}-\lambda_{1}\right) f_{1}^{0},\left(b_{2}-\lambda_{2}\right) f_{2}^{\infty}\right)(x)\right| \\
& \leq C \sum_{l=1}^{\infty} \frac{1}{\left|2^{l+1} Q\right|^{2}} \prod_{j=1}^{2}\left(\int_{2^{l+1} Q}\left|f_{j}\left(y_{j}\right)\right|^{p_{j}} \omega_{j}\left(y_{j}\right) d y_{j}\right)^{1 / p_{j}} \\
& \quad \times\left(\int_{2^{l+1} Q}\left|b_{j}\left(y_{j}\right)-\lambda_{j}\right|^{p_{j}^{\prime}} \omega_{j}\left(y_{j}\right)^{-p_{j}^{\prime} / p_{j}} d y_{j}\right)^{1 / p_{j}^{\prime}} \\
& \leq C \prod_{j=1}^{2}\left\|f_{j}\right\|_{L^{p_{j}, k}\left(\omega_{j}\right)} \sum_{l=1}^{\infty} l^{2} v_{\vec{\omega}}\left(2^{l+1} Q\right)^{(k-1) / p} .
\end{aligned}
$$

So,

$$
\left(\frac{1}{v_{\vec{\omega}}(Q)^{k}} \int_{Q}\left|I I_{4}\right|^{p} v_{\vec{\omega}}(x) d x\right)^{1 / p} \leq C \prod_{j=1}^{2}\left\|b_{j}\right\|_{B M O}\left\|f_{j}\right\|_{L^{p_{j}, k}\left(\omega_{j}\right)} .
$$

Finally, we still split $T_{\Pi \mathbf{b}}\left(f_{1}^{\infty}, f_{2}^{\infty}\right)(x)$ into four terms:

$$
\begin{aligned}
T_{\Pi \mathbf{b}}\left(f_{1}^{\infty}, f_{2}^{\infty}\right)(x) \\
=\left(b_{1}(x)-\lambda_{1}\right)\left(b_{2}(x)-\lambda_{2}\right) T\left(f_{1}^{\infty}, f_{2}^{\infty}\right)(x)-\left(b_{1}(x)-\lambda_{1}\right) T\left(f_{1}^{\infty},\left(b_{2}-\lambda_{2}\right) f_{2}^{\infty}\right)(x) \\
\quad-\left(b_{2}(x)-\lambda_{2}\right) T\left(\left(b_{1}-\lambda_{1}\right) f_{1}^{\infty}, f_{2}^{\infty}+T\left(\left(b_{1}-\lambda_{1}\right) f_{1}^{\infty},\left(b_{2}-\lambda_{2}\right) f_{2}^{\infty}\right)(x)\right)(x) \\
=I V_{1}+I V_{2}+I V_{3}+I V_{4} .
\end{aligned}
$$

Because each term of $I V_{j}$ is completely analogous to $I I_{j}, j=1,2,3,4$ with a small difference, we only estimate $I V_{1}$ :

$$
\begin{aligned}
\left|T\left(f_{1}^{\infty}, f_{2}^{\infty}\right)(x)\right| & \leq C \int_{\left(\mathbb{R}^{n}\right)^{2} \backslash(2 Q)^{2}} \frac{\left|f_{1}\left(y_{1}\right) f_{2}\left(y_{2}\right)\right|}{\left(\left|x-y_{1}\right|+\left|x-y_{2}\right|\right)^{2 n}} d y_{2} d y_{1} \\
& \leq C \sum_{l=1}^{\infty} \int_{\left(2^{l+1} Q\right)^{2} \backslash\left(2^{l} Q\right)^{2}} \frac{\left|f_{1}\left(y_{1}\right) f_{2}\left(y_{2}\right)\right|}{\left(\left|x-y_{1}\right|+\left|x-y_{2}\right|\right)^{2 n}} d y_{2} d y_{1} \\
& \leq C \sum_{l=1}^{\infty} \frac{1}{\left|2^{l+1} Q\right|^{2}} \int_{\left(2^{l+1} Q\right)^{2}} \prod_{j=1}^{2}\left|f_{j}\left(y_{j}\right)\right| d y_{j} \\
& \leq C \prod_{j=1}^{2}\left\|f_{j}\right\|_{L^{p_{j}, k}\left(\omega_{j}\right)} \sum_{l=1}^{\infty} v_{\widehat{\omega}}\left(2^{l+1} Q\right)^{(k-1) / p} .
\end{aligned}
$$


Hence,

$$
\left(\frac{1}{v_{\vec{\omega}}(Q)^{k}} \int_{Q}\left|I V_{1}\right|^{p} v_{\vec{\omega}}(x) d x\right)^{1 / p} \leq C \prod_{j=1}^{2}\left\|b_{j}\right\|_{B M O}\left\|f_{j}\right\|_{L^{p_{j}, k}\left(\omega_{j}\right)}
$$

Combining all estimates, we complete the proof of Theorem 1.1.

We now turn to the proof of Theorem 1.2.

Proof of Theorem 1.2 By homogeneity, we may assume that $\lambda=\left\|b_{1}\right\|_{B M O}=\left\|b_{2}\right\|_{B M O}=1$ and we only need to prove that

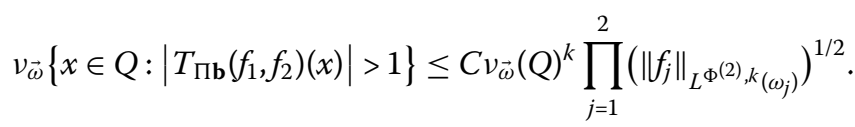

To prove the above inequality, we can write

$$
\begin{aligned}
v_{\vec{\omega}} & \left\{x \in Q:\left|T_{\Pi \mathbf{b}}\left(f_{1}, f_{2}\right)(x)\right|>1\right\} \\
\leq & v_{\vec{\omega}}\left\{x \in Q:\left|T_{\Pi \mathbf{b}}\left(f_{1}^{0}, f_{2}^{0}\right)(x)\right|>1 / 4\right\}+v_{\vec{\omega}}\left\{x \in Q:\left|T_{\Pi \mathbf{b}}\left(f_{1}^{0}, f_{2}^{\infty}\right)(x)\right|>1 / 4\right\} \\
& \quad+v_{\vec{\omega}}\left\{x \in Q:\left|T_{\Pi \mathbf{b}}\left(f_{1}^{\infty}, f_{2}^{0}\right)(x)\right|>1 / 4\right\}+v_{\vec{\omega}}\left\{x \in Q:\left|T_{\Pi \mathbf{b}}\left(f_{1}^{\infty}, f_{2}^{\infty}\right)(x)\right|>1 / 4\right\} \\
= & V+V I+V I I+V I I I
\end{aligned}
$$

for any cube Q. Employing Lemma 2.9 and Lemma 2.2, we have

$$
\begin{aligned}
V & \leq C \prod_{j=1}^{2}\left(\int_{\mathbb{R}^{n}} \Phi^{(m)}\left(\left|f_{j}(x)\right|\right) \omega_{j}(x) d x\right)^{1 / 2} \\
& \leq C \prod_{j=1}^{2}\left[\omega_{j}(Q)^{k}\left\|f_{j}\right\|_{L^{\Phi^{(m)}, k}\left(\omega_{j}\right)}\right]^{1 / 2} \\
& \leq C v_{\vec{\omega}}(Q)^{k} \prod_{j=1}^{2}\left[\left\|f_{j}\right\|_{L^{\Phi(m), k}\left(\omega_{j}\right)}\right]^{1 / 2} .
\end{aligned}
$$

From Lemma 2.6 and Lemma 2.4, we deduce that

$$
\begin{aligned}
& v_{\vec{\omega}}\left\{x \in Q:\left|T_{\Pi \mathbf{b}}\left(f_{1}^{0}, f_{2}^{\infty}\right)(x)\right|>1 / 4\right\} \\
& \quad \leq \sup _{t>0} \frac{1}{\Phi^{(m)}\left(\frac{1}{t}\right)} v_{\vec{\omega}}\left\{x \in Q:\left|T_{\Pi \mathbf{b}}\left(f_{1}^{0}, f_{2}^{\infty}\right)(x)\right|>t^{2}\right\} \\
& \leq C_{v_{\vec{\omega}}, \mathbf{b}} \sup _{t>0} \frac{1}{\Phi^{(m)}\left(\frac{1}{t}\right)} v_{\vec{\omega}}\left(\left\{y \in Q:\left|\mathcal{M}_{\Phi}\left(f_{1}^{0}, f_{2}^{\infty}\right)(y)\right|>t^{2}\right\}\right) \\
& \leq C_{v_{\vec{\omega}}, \mathbf{b}} \sup _{t>0} \frac{1}{\Phi^{(m)}\left(\frac{1}{t}\right)} v_{\vec{\omega}}\left(\left\{y \in Q:\left|M_{\Phi}\left(f_{1}^{0}\right)(y) M_{\Phi}\left(f_{2}^{\infty}\right)(y)\right|>t^{2}\right\}\right) \\
& \leq \frac{C_{v_{\vec{\omega}}, \mathbf{b}}}{t}\left(\int_{\mathbb{R}^{n}} \Phi\left(\left|f_{1}^{0}\right|\right)(y) M\left(\chi_{Q} \omega_{1}\right)(y) d y \int_{\mathbb{R}^{n}} \Phi\left(\left|f_{2}^{\infty}\right|\right)(y) M\left(\chi_{Q} \omega_{2}\right)(y) d y\right)^{1 / 2} \\
& \leq \frac{C_{v_{\vec{\omega}}, \mathbf{b}}}{t}\left[\omega_{j}(Q)^{k} \mid f_{j} \|_{L^{\Phi, k}\left(\omega_{j}\right)}\right]^{1 / 2},
\end{aligned}
$$


where the last inequality holds by the (3.10) and (3.11) in [15]. Then from Lemma 2.2 and the fact that $t \Phi\left(\frac{1}{t}\right)>1$, we have

$$
V I \leq C v_{\bar{\omega}}(Q)\left[\omega_{j}(Q)^{k}\left\|f_{j}\right\|_{L^{\Phi, k}\left(\omega_{j}\right)}\right]^{1 / 2} .
$$

A similar statement follows:

$$
\begin{aligned}
& V I I \leq C v_{\vec{\omega}}(Q)\left[\omega_{j}(Q)^{k}\left\|f_{j}\right\|_{L^{\Phi, k}\left(\omega_{j}\right)}\right]^{1 / 2} ; \\
& V I I I \leq C v_{\vec{\omega}}(Q)\left[\omega_{j}(Q)^{k}\left\|f_{j}\right\|_{L^{\Phi, k}\left(\omega_{j}\right)}\right]^{1 / 2} .
\end{aligned}
$$

Thus we complete the proof of Theorem 1.2.

\section{A problem}

Fix $N \in \mathbb{N}$. Let $m \in C^{L}\left(\mathbb{R}^{N n} \backslash\{0\}\right)$, for some positive integer $L$, satisfying the following condition:

$$
\left|\partial_{\xi_{1}}^{\alpha_{1}} \cdots \partial_{\xi_{N}}^{\alpha_{N}} m\left(\xi_{1}, \ldots, \xi_{N}\right)\right| \leq C_{\alpha_{1}, \ldots, \alpha_{N}}\left(\left|\xi_{1}\right|+\cdots+\left|\xi_{N}\right|\right)^{|\alpha|}
$$

for all $|\alpha| \leq s$ and $\xi \in \mathbb{R}^{N n} \backslash\{0\}$, where $\alpha=\left(\alpha_{1}, \ldots, \alpha_{N}\right)$ and $\xi=\left(\xi_{1}, \ldots, \xi_{N}\right)$. The multilinear Fourier multiplier operator $T_{N}$ is defined by

$$
T_{m}(\vec{f})(x)=\frac{1}{(2 \pi)^{N n}} \int_{\left(\mathbb{R}^{N n}\right)} e^{i x\left(\xi_{1}+\cdots+\xi_{N}\right)} m\left(\xi_{1}, \ldots, \xi_{N}\right) \hat{f}_{1}\left(\xi_{1}\right) \cdots \hat{f}_{N}\left(\xi_{N}\right) d \xi_{1} \cdots d \xi_{N}
$$

for all $f_{1}, \ldots, f_{N} \in \mathcal{S}\left(\mathbb{R}^{n}\right)$, where $\vec{f}=\left(f_{1}, \ldots, f_{N}\right)$. If $\mathcal{F}^{-1} m$ is an integrable function, then this can also be written as

$$
T_{m}(\vec{f})(x)=\int_{\left(\mathbb{R}^{N n}\right)} \mathcal{F}^{-1} m\left(x-y_{1}, \ldots, x-y_{N}\right) f\left(y_{1}\right) \cdots f\left(y_{N}\right) d y_{1} \cdots d y_{N}
$$

In [18], Fujita and Tomita obtained the following theorem.

Theorem 4.1 Let $1<p_{1}, \ldots, p_{N}<\infty, \frac{1}{p_{1}}+\cdots+\frac{1}{p_{N}}=\frac{1}{p}$ and $\frac{n}{2}<s_{j} \leq n$ for $1 \leq j \leq N$. Assume $p j>n / s j$ and $w_{j} \in A_{p_{j} s_{j} / n}$ for $1 \leq j \leq N$. If $m \in L^{\infty}\left(\mathbb{R}^{N n}\right)$ satisfies

$$
\left\|m_{k}\right\|_{W^{\left(s_{1}, \ldots, s_{N}\right)}}=\left(\int_{\mathbb{R}^{N n}}\left(1+\left|\xi_{1}\right|^{2}\right)^{1 / 2} \cdots\left|\left(1+\left|\xi_{N}\right|^{2}\right)^{1 / 2} \hat{m}(\xi)\right|^{2} d \xi\right)^{1 / 2}<\infty
$$

then $T_{N}$ is bounded from $L^{p_{1}}\left(\omega_{1}\right) \times \cdots \times L^{p_{N}}\left(\omega_{N}\right)$ to $L^{p}\left(v_{\vec{\omega}}\right)$, where

$$
m_{j}(\xi)=m\left(2^{j} \xi_{1}, \ldots, 2^{j} \xi_{N}\right) \Psi\left(\xi_{1}, \ldots \xi_{N}\right)
$$

where $\Psi$ is the Schwarz function and satisfies

$$
\operatorname{supp} \Psi \subset\left\{\xi \in \mathbb{R}^{N n}: 1 / 2 \leq|\xi| \leq 2\right\}, \quad \sum_{k \in \mathbb{Z}} \Psi\left(\xi / 2^{k}\right)=1 \quad \text { for all } \xi \in \mathbb{R}^{N n} \backslash\{0\} .
$$


A natural problem is whether the Lebesgue spaces $L^{p_{j}}\left(\omega_{j}\right)$ and $L^{p}\left(v_{\vec{\omega}}\right)$ can be replaced by $L^{p_{j}, k}(\omega)$ and $L^{p, k}\left(v_{\vec{\omega}}\right)$. It should be pointed out that the method in this paper may not be suitable to address this problem.

\section{Competing interests}

The authors declare that they have no competing interests.

\section{Authors' contributions}

All authors contributed equally to the writing of this paper. All authors read and approved the final manuscript.

\section{Acknowledgements}

The authors would like to thank the referee for some very valuable suggestions. This research was supported by NSF of China (no. 11161044, no. 11261055) and by XJUBSCX-2012004

Received: 6 September 2013 Accepted: 18 February 2014 Published: 04 Mar 2014

\section{References}

1. Coifman, R, Meyer, Y: On commutators of singular integral and bilinear singular integrals. Trans. Am. Math. Soc. 212, 315-331 (1975)

2. Grafakos, L, Torres, R: Multilinear Calderón-Zygmund theory. Adv. Math. 165, 124-164 (2002)

3. Grafakos, L, Torres, R: On multilinear singular integrals of Calderón-Zygmund type. Publ. Mat. Extra, 57-91 (2002)

4. Duong, XT, Grafakos, L, Yan, L-X: Multilinear operators with non-smooth kernels and commutators of singular integrals. Trans. Am. Math. Soc. 362, 2089-2113 (2010)

5. Duong, XT, Gong, R, Grafakos, L, Li, J, Yan, L-X: Maximal operator for multilinear singular integrals with non-smooth kernels. Indiana Univ. Math. J. 58, 2517-2541 (2009)

6. Komori, Y, Shirai, S: Weighted Morrey spaces and a singular integral operator. Math. Nachr. 282(2), 219-231 (2002)

7. Lerner, AK, Ombrosi, S, Pérez, C, Torres, RT, Trujillo-González, R: New maximal functions and multiple weights for the multilinear Calderón-Zygmund theory. Adv. Math. 220, 1222-1264 (2009)

8. Grafakos, L, Torres, R: Maximal operator and weighted norm inequalities for multilinear singular integrals. Indiana Univ. Math. J. 51, 1261-1276 (2002)

9. Pérez, C, Torres, RH: Sharp maximal function estimates for multilinear singular integrals. Contemp. Math. 320, 323-331 (2003)

10. Pérez, C, Pradolini, G, Torres, RH, Trujillo-González, RT: End-point estimates for iterated commutators for multilinear singular integrals. arXiv:1004.4976v2 [math.CA] (2011)

11. Pérez, C, Trujillo-González, R: Sharp weighted estimates for multilinear commutators. J. Lond. Math. Soc. 65, 672-692 (2002)

12. Ding, Y, Yang, D-C, Zhou, Z: Boundedness of sublinear operators and commutators on $L_{\omega}^{p}\left(\mathbb{R}^{n}\right)$. Yokohama Math. J. 46, 15-27 (1998)

13. Lu, S-Z, Yang, D-C, Zhou, Z: Sublinear operators with rough kernel on generalized Morrey spaces. Hokkaido Math. J. 27, 219-232 (1998)

14. Shi, S-G, Fu, Z-W, Zhao, F-Y: Estimates for operators on weighted Morrey spaces and their applications to nondivergence elliptic equations. J. Inequal. Appl. 2013, 390 (2013)

15. Ye, X: Some estimates for multilinear commutators on the weighted Morrey spaces. Math. Sci. 6, 33 (2012). http://www.iaumath.com/content/6/1/33

16. Wang, H, Yi, W: Multilinear singular and fractional integral operators on weighted Morrey spaces. arXiv:1303.4480v1 [math.CA] (2013)

17. Muckenhoupt, B, Wheeden, R: Weighted bounded mean oscillation and the Hilbert transform. Stud. Math. 54, 221-237 (1976)

18. Fujita, M, Tomita, N: Weighted norm inequalities for multilinear Fourier multiplier. Trans. Am. Math. Soc. (2012). doi:10.1090/S0002-9947-2012-05700-X 